\title{
Study on the Mechanism of Harmonic Amplification of Cable Leakage capacitance Based on MATLAB
}

\author{
Xiaofei Chen ${ }^{\mathrm{a}}$, Zejing $\mathrm{Qiu}^{\mathrm{b}}$ and Chupeng Xiao ${ }^{\mathrm{c}}$ \\ Nari (Wuhan) electrical equipment \&engineering efficiency evaluation center, Wuhan 430074, \\ China \\ axiaofei2011wd@163.com, b283236661@qq.com, c181259409@qq.com
}

Keywords: Harmonic impedance, harmonic amplification, leakage capacitance, resonance.

\begin{abstract}
Taking the distribution cable as the research object, based on the circuit theory, the equivalent circuit parameters of the cable are calculated. On the basis of this, the harmonic impedance model of distribution cable is established, The amplification mechanism of the leakage capacitance of the cable is analyzed, and the change curve of the harmonic current amplification factor with the cable length and the frequency of harmonic wave is obtained by MATLAB simulation. From the curve can be drawn, the curve of the extreme point is the parallel resonant point. In this paper, the analysis of harmonic current amplification mechanism and the harmonic impedance model of distribution cable is of some guiding significance to avoid the resonance of the cable line.
\end{abstract}

\section{Introduction}

With the rapid increase of urban electricity consumption, the application of cable has been greatly developed because of the advantages that the overhead line does not possess. Cable buried in the ground, the capacitance of the cable to earth is about $10 \sim 20$ times that of the overhead transmission line, but the inductance of cable is about 1/2 1/3 overhead transmission line [1]. In the presence of harmonics, the resonance problem of the cable line will lead to the increase of the cable current carrying capacity, in particular, the amplification of harmonic current leads to the increase of line loss, which leads to serious heating of the cable and accelerate cable aging [2].

At home and abroad, most of the researches on the modeling of overhead lines, there is less research on the cables. In paper [3], the different calculation methods of cable electrical parameters are studied and compared, however, the influence of harmonics on the impedance characteristics of the cable is not considered; in paper [4] studies the harmonic amplification mechanism of power supply system of electric railway, but the electrical characteristic of the cable is different from that of overhead transmission line, need further study.

In order to study the mechanism of harmonic current amplification, in this paper, based on the circuit theory and the unit equivalent model of transmission line, the unit equivalent circuit of distribution cable is established, on this basis, the harmonic equivalent model of distribution cable is established, the harmonic current amplification curve is obtained by simulation analysis.

\section{Distribution Cable Harmonic Impedance Model}

\subsection{Distribution Cable Unit Equivalent Circuit.}

There are four main electrical parameters of cable line: resistance, inductance, conductance and capacitance. Considering the cross-section of cable and the power supply characteristics of cable, based on the circuit theory, the equivalent circuit of the cable is established as follows in figure 1. 


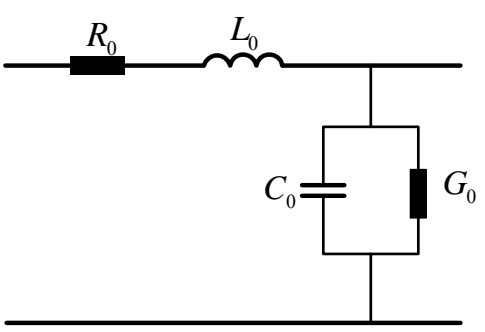

Fig. 1 Equivalent circuit of distribution cable unit length

It is assumed that the parameters of transmission cable line are uniformly distributed, is the unit length resistance of the cable, is the unit length inductance, is the unit length capacitance, and the is the conductance per unit length.

\subsection{Calculation of Equivalent Circuit Parameters of Distribution Cable.}

For distribution cables, the formula for calculating the inductance per unit length is as follows:

$$
L=0.05+0.2 \times \ln \frac{k s}{r}(m H / k m)
$$

Formula (1), $\mathrm{k}$ - coefficient, for parallel structural cables, $\mathrm{k}=1.26$; s- the distance between the center conductor $(\mathrm{mm}), \mathrm{r}$ - conductor radius.

Through the formula (1) calculation, it is concluded that the inductance value of the cable line is in the range of $0.1 \sim 0.7 \mathrm{mH} / \mathrm{km}$, because the inductance value is small, it can be regarded as a short circuit in the calculation.

Considering the skin effect and proximity effect, active loss of AC current in conductors is greater than that of DC current, so the AC resistance in the cable is larger than the DC resistance, calculation formula of cable resistance is given below [5].

$$
R_{a c}=R_{d c}\left(1+y_{s}+y_{p}\right)
$$

$y_{s}$ is the skin effect factor; $y_{p}$ is the proximity effect factor; $R_{d c}$ is the DC resistance of the conductor at a given temperature; $R_{a c}$ is the $\mathrm{AC}$ resistance of the conductor at a given temperature.

$$
R_{a c}=\rho \frac{L}{S}
$$

In formula (2), $\mathrm{p}$ - resistivity; $\mathrm{L}$ - cable length (m): $\mathrm{S}$ - cross-sectional area of the cable core $\left(\mathrm{mm}^{2}\right)$.

The formula to calculate the leakage capacitance on the shielding layer of cable can be expressed as [6]:

$$
C_{0}=\frac{0.0056 \varepsilon}{\ln \frac{d_{2}}{d_{1}}} \quad(\mu \mathrm{F} / \mathrm{km})
$$

Formula (4), $\varepsilon$--dielectric constant of dielectric in cable, $d_{1}$--cable core diameter, $d_{2}$--diameter of the cable insulation layer.

Using the formula (3), the calculation results show that the leakage capacitance of the cable is in the range of $0.05 \sim 0.75 \mathrm{~F}$, and the number of the cable to earth resistance is of magnitude of 109 , can be regarded as open circuit.

Through the calculation of the equivalent circuit parameters of power distribution cables, considering the main harmonic number in distribution system is 2 50 times, when establishing the harmonic impedance model of distribution cable, we can ignore the ground resistance of the cable and the cable inductance[7], while the leakage capacitance of the cable is taken as the main parameter. Gives the equivalent circuit of the cable impedance as shown in Figure 2: 


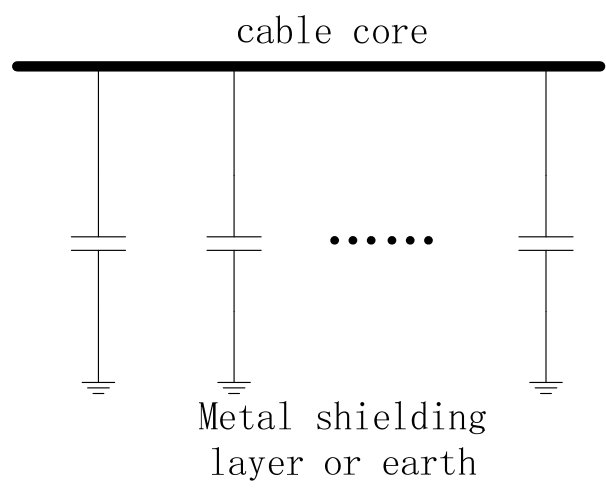

Fig. 2 Schematic diagram of harmonic impedance of power distribution cable

\subsection{The Influence of Leakage Capacitance on System Harmonic Impedance}

If the leakage capacity of the distribution cable is not considered, and the power supplied to power loading (inductive) by the cable, the impedance expression of the cable circuit in the fundamental wave is

$$
Z_{l}=R_{l}+j X_{l}
$$

In formula (5), $X_{l}=j \omega_{0} L, \omega_{0}=2 \pi f_{0}, f_{0}=50 \mathrm{~Hz}$.

In the system, when there is $\mathrm{n}$ subharmonic, the reactance of the $\mathrm{N}$ harmonic is $X_{l_{n}}=n X_{l}$, resistance is $R_{l_{n}}=R_{l}$, the $\mathrm{N}$ harmonic impedance is expressed as[8]:

$$
Z_{l_{n}}=R_{l_{n}}+j X_{l_{n}}=\left|Z_{l_{n}}\right| e^{j \theta_{l_{n}}}
$$

With the increase of the length of distribution cable, the value of leakage capacitance becomes larger, capacitive character of the line enhanced, so when calculating the cable system impedance model, the cable to ground leakage capacitance must be taken into account [9]. Figure 3 is the impedance model of the harmonic cable when considering the leakage capacitance.

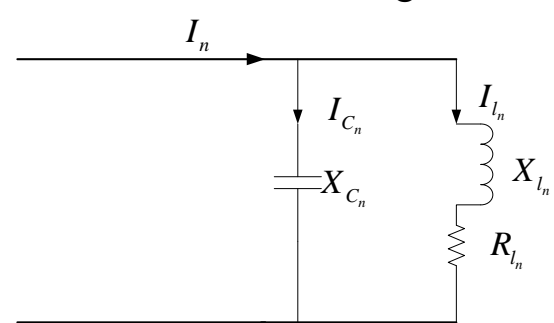

Fig. 3 Harmonic impedance model of cable line considering leakage capacitance

Assuming the fundamental reactance of the leakage capacitor is $X_{C}$, the reactance under the $\mathrm{N}$ harmonic is $X_{C_{n}}=\frac{1}{n} X_{C}$, the input impedance of the cable line in consideration of the leakage capacitance is as follows:

$$
Z_{l_{n}}^{\prime}=\frac{-j X_{C_{n}} Z_{l_{n}}}{R_{l_{n}}+j\left(X_{l_{n}}-X_{C_{n}}\right)}=\left|Z_{l_{n}}^{\prime}\right| e^{j \theta_{l_{n}}^{\prime}}
$$

From formula (6) formula (7) can be drawn, under the influence of the leakage capacitance of the cable, the harmonic impedance of the system changes, when the parameters match, the parallel resonance will occur, so that the harmonic impedance of the system reaches the maximum.

\section{Analysis of Harmonic Current Amplification in Distribution Cable}

In the power system, such as rectifier equipment, electric arc furnace harmonic sources can be equivalent to the constant current source, that is, when the external harmonic impedance changes, the harmonic current in the input network can be assumed to be constant. When inductive loads (such as asynchronous motor) are powered by cables, there are harmonic sources near (As shown in Figure 4), 
considering the capacitive impedance of the cable, the current into cable leakage capacitance and inductive load will change with the number of harmonics [10].

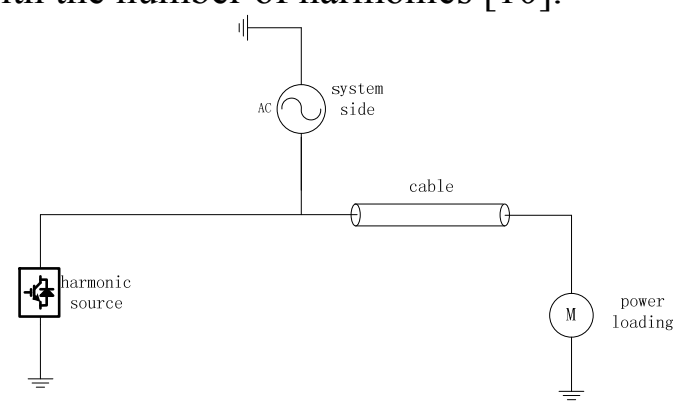

Fig. 4 Cable power supply system diagram

Draw the equivalent circuit diagram of Figure 4 as shown in Figure 5, Among them, $X_{s}$ and $R_{s}$ are the fundamental equivalent reactance and equivalent resistance of the system side, $X_{l}$ and $R_{l}$ are the fundamental equivalent reactance and equivalent resistance of the load side, $I_{n}$ is $n$ harmonic current output by harmonic source, $I_{l_{n}}$ is the current into the electrical equipment, $I_{C_{n}}$ is the leakage capacitance current.

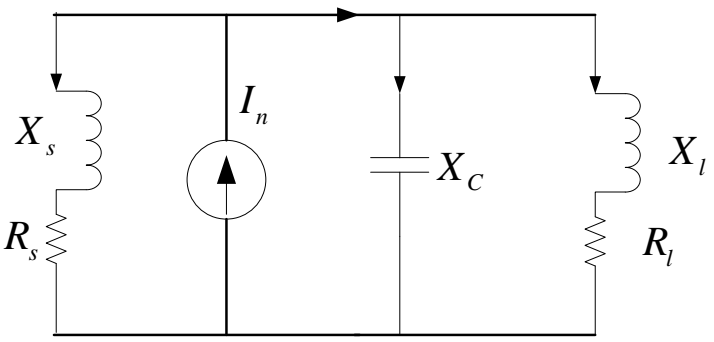

Fig. 5 Equivalent circuit diagram of cable power supply system

The system impedance and load impedance are calculated in parallel, the equivalent circuit diagram of the cable power supply system is reduced to figure 6 .

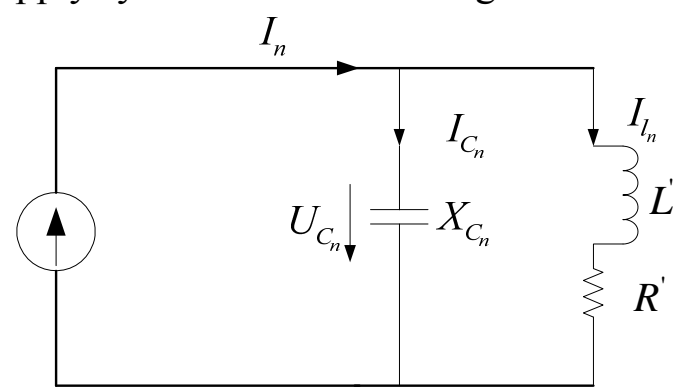

Fig. 6 Simplified diagram of equivalent circuit of cable power supply system

In the figure,

$$
\begin{aligned}
& R^{\prime}=\frac{\left(R_{s} R_{l}-\omega^{2} L_{s} L_{l}\right)\left(R_{s}+R_{l}\right)+\omega^{2}\left(L_{s} R_{l}+L_{l} R_{s}\right)\left(L_{s}+L_{l}\right)}{\left(R_{s}+R_{l}\right)^{2}+\omega^{2}\left(L_{s}+L_{l}\right)^{2}} \\
& L^{\prime}=\frac{\left(L_{s} R_{l}+L_{l} R_{s}\right)\left(R_{s}+R_{l}\right)-\left(R_{l} R_{s}-\omega^{2} L_{s} L_{l}\right)\left(L_{s}+L_{l}\right)}{\left(R_{s}+R_{l}\right)^{2}+\omega^{2}\left(L_{s}+L_{l}\right)^{2}} \\
& \dot{I_{C_{n}}}=\frac{R_{l_{n}}+j X_{l_{n}}}{R_{l_{n}}+j\left(X_{l_{n}}-X_{C_{n}}\right)} \dot{I}_{n}=\frac{R_{l}+j n X_{l}}{R_{l}+j\left(n X_{l}-\frac{1}{n} X_{C}\right)} \dot{I_{n}} \\
& \dot{I_{l_{n}}}=\frac{-j X_{C_{n}}}{R_{l_{n}}+j\left(X_{l_{n}}-X_{C_{n}}\right)} \dot{I_{n}}=\frac{-j \frac{1}{n} X_{C}}{R_{l}+j\left(n X_{l}-\frac{1}{n} X_{C}\right)} \dot{I_{n}}
\end{aligned}
$$




$$
\dot{U_{C_{n}}}=-j \dot{I_{C_{n}}} X_{C_{n}}=-j \frac{R_{l}+j n X_{l}}{R_{l}+j\left(n X_{l}-\frac{1}{n} X_{C}\right)} \dot{I_{n}} \cdot \frac{1}{n} X_{C}
$$

When the harmonic current frequency of the inductor and capacitor branches satisfies the resonant condition of inductance and capacitance, that is $n X_{l}-\frac{1}{n} X_{C}=0$, the harmonic current value of the two branch of harmonic current will greatly exceed injected harmonic current $I_{n}$, that is, the phenomenon of harmonic current amplification will occur[11,12].

The following are the definitions of the harmonic current amplification factor of the cable leakage capacitor branch and inductance branch, respectively, $\beta_{c}$ and ${ }^{\beta_{l}}$ :

$$
\begin{gathered}
\beta_{c}=\frac{\dot{I_{C_{n}}}}{\dot{I_{n}}}=\frac{R_{l_{n}}+j X_{l_{n}}}{R_{l_{n}}+j\left(X_{l_{n}}-X_{C_{n}}\right)}=\frac{R_{l}+j n X_{l}}{R_{l}+j\left(n X_{l}-\frac{1}{n} X_{C}\right)} \\
\beta_{l}=\frac{\dot{I_{l_{n}}}}{\dot{I_{n}}}=\frac{-j X_{C_{n}}}{R_{l_{n}}+j\left(X_{l_{n}}-X_{C_{n}}\right)}=\frac{-j \frac{1}{n} X_{C}}{R_{l}+j\left(n X_{l}-\frac{1}{n} X_{C}\right)}
\end{gathered}
$$

For a $10 \mathrm{kV}$ distribution network, the system impedance is $0.99 \Omega$, the impedance of the induction motor fed by a power cable of length $\mathrm{L}$ is $Z_{m}=R_{m}+\mathrm{jX} \mathrm{m}_{m}=(2.2+3.768 \mathrm{j}) \Omega$, considering that the resistance in the system impedance is much smaller than that of the reactance, which can be ignored, that is $Z_{S}=R_{S}+j X_{S} \approx j X_{S}$, then can be calculated that $L_{S}=0.0032 \mathrm{H}, L_{m}=0.012 \mathrm{H}$. The formula (8) and formula (9) can be calculated: $R^{\prime}=0.0762 \Omega, L^{\prime}=0.0031 \mathrm{H}, X_{l}=0.9734 \Omega$.

Select the nominal section of the $240 \mathrm{~mm}$ JLV-6/10KV - Single core XLPE power cable as an example, $=17.5 \mathrm{~mm}=34.9 \mathrm{~mm},=2.5$.

Using the formula (4), the cable leakage capacity at the unit length is calculated, $C_{0}=0.20 \mu \mathrm{F} / \mathrm{km}$, the leakage capacitance of the distribution cable with a length of $\mathrm{Lkm}$ is $C_{l}=0.2 L \mu \mathrm{F}$. Use the formula:

$$
\begin{aligned}
& \beta_{c}=\frac{\dot{I_{C_{n}}}}{\dot{I_{n}}}=\frac{R_{l}+j n X_{l}}{R_{l}+j\left(n X_{l}-\frac{1}{n} X_{C}\right)}=\frac{0.0762+0.9734 n j}{0.0762+0.9734 n j-\frac{15.78}{n L} j} \\
& \left|\beta_{c}\right|=\sqrt{\frac{0.06+0.95 n^{2}}{0.06+0.95 n^{2}-31000.84 / l+15924^{2} / l^{2} n^{2}}}
\end{aligned}
$$

Analysis Based on MATLAB, take harmonic number $\mathrm{n}$ from fundamental to the 50 harmonic, distribution cable length varies from $10 \mathrm{~km}$ to $60 \mathrm{~km}$, and the simulation results are as follows: 


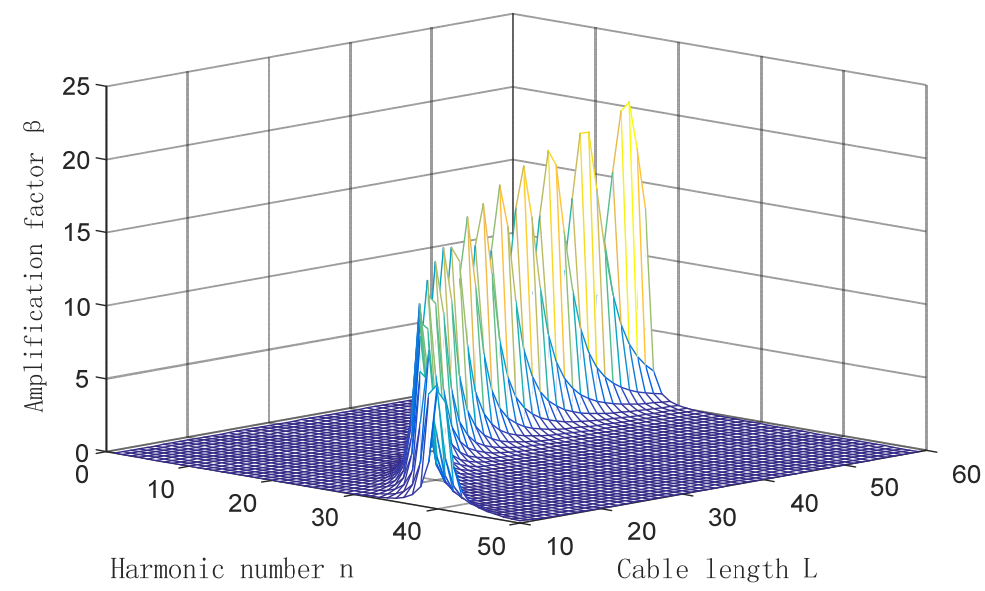

Fig. 7 Variation curve of current amplification factor

By analyzing the simulation results, the maximum value of the amplification factor is at the resonance point, i.e. $n X_{l}-\frac{1}{n} X_{C}=0$, in the case of cable length determination, the cable leakage capacitance can be determined, resonance frequency can be obtained, $n=\sqrt{\frac{X_{C}}{X_{l}}}$, at this time, the leakage current in the capacitor will be several dozen times higher than that of the original harmonic current, the voltage at the two ends of the leakage capacitor will reach the maximum value, due to the resonance frequency is relatively high, the harmonic voltage will be very large. If the distribution cable is in a state of harmonic current amplification for a long time, it will lead to the cable temperature rise, and even cause a fire, at the same time, because of the increase of the impedance of the system caused by harmonics, the harmonic current will lead to the increase of the line loss, reduce the energy efficiency of the network, and accelerate the aging of the cable insulation.

\section{Summary}

In this paper, based on the basic theory of circuit theory, the unit equivalent circuit of distribution cable is established based on the method of unit equivalent circuit modeling of overhead transmission lines, based on the calculation of the four main electrical parameters of the cable line, the unit length resistance, inductance, capacitance and conductance, the harmonic impedance model of the distribution cable is established. Through the comparison and analysis of the four main electrical parameters, it is concluded that the main characteristics of the distribution cable are the capacitive impedance and the leakage capacitance of the cable. The harmonic current amplification mechanism of distribution cable is analyzed, that is to say, when the leakage capacitance of the cable and the system impedance occur parallel resonance in a certain harmonic current, harmonic current amplification occurs, the analysis of harmonic current amplification mechanism and the harmonic impedance model of distribution cable is of some guiding significance to avoid the resonance of the cable line.

\section{References}

[1]. Nasser D. Tleis. Power systems modeling and fault analysis. Elsevier Ltd.2008:140-174.

[2]. Galloway, R.H., et al., Calculation of electrical parameters for short and long polyphaser transmission lines, Proceedings IEE, Vol. Ill, December1964, and 2051-2059.

[3]. XU Zheng, QIAN Jie. Comparison of Different Methods for Calculating Electrical Parameters of Power Cables [J].High Voltage Engineering, 2013, 39(3):689-697. 
[4]. Li Qionglin, Li Che nying, et al. Impact analysis and calculation method of harmonic on power cable loss [J]. Electrical Measurement \& Instrumentation, 2015, 52(23):34-42.

[5]. Jean Carlos Hernández Mejia. Characterization of Real Power Cable Defects by Diagnostic Measurements [D]. Georgia Institute of Technology.2008.

[6]. Incropera. Calculations of Temperature Distributions within Cable Trench Backfill and the Surrounding Soil [J]. IEEE Trans on Apparatus and Systems, 1985, 3(8):1973-1977

[7]. D. Dubois, P. Mirebeau: The use of insulated wires Milliken conductors in high voltage power transmission underground AC lines, Proc. of JICABLE2007:488-493.

[8]. R. Benato, A. Paolucci: Operating Capability of Long AC EHV Transmission Cables, Electric Power Systems Research, Vol. 75/1, July 2005, pp. 17-27.

[9]. G.J. Anders: Rating of electric power cables in unfavorable thermal environment, IEEE Press, Wiley-Interscience, New Jersey, 2005.

[10]. G. C. Montanari, et al. searching for the factors which affect self-healing capacitor degradation under non-sinusoidal voltage. IEEE Trans. on Dielectrics and Electrical Insulation, 1999, 6(3):319-325.

[11]. Hirofumi Akagi etal. A New Power line Conditioner for Harmonic Compensation in Power System. IEEE ICHPS VI 1994 Bologna, Italy.

[12]. ARNOLD, A.H.M.: 'Eddy-current losses in multi-core paper-insulated lead-covered cables, armored and unarmored, carrying balanced 3-phase current', J.IEE, 1942, 8911, pp.639-647. 